\title{
Multidimensional "Paperfolding" Structures Three and Four Dimensions
}

\author{
S.I. Ben-Abraham ${ }^{a, *}$, D. Flom ${ }^{a}$, R. Richman ${ }^{a}$ And D. Shapira ${ }^{b}$ \\ ${ }^{a}$ Department of Physics, Ben-Gurion University of the Negev, POB 653, IL-84105 Beer-Sheva, Israel \\ ${ }^{b}$ The Racah Institute of Physics, The Hebrew University, IL-91904 Jerusalem, Israel
}

\begin{abstract}
We recapitulate our previously developed recursive algorithm for creating "paperfolding" structures in arbitrary dimensions. Here we explain and apply it specifically to three and four dimensions. We visualize the results by suitable projections. We also explicitly enumerate the number of "folds" in the first four generations of the recursion. We conjecture (without proof) that the Fourier spectrum of the structures is pure point (the Bragg peaks).
\end{abstract}

DOI: 10.12693 /APhysPolA.126.435

PACS: $61.44 . \mathrm{Br}$

\section{Introduction}

The paperfolding sequences were originally discovered by actually folding repeatedly a sheet of paper resulting in two kinds of folds called crests and valleys or positive and negative, respectively. This nice little game became fast the subject of serious research and thus the regular paperfolding sequence turned out to be one of the standard paradigmatic almost periodic structures. Most the properties of the regular paperfolding sequence are shared also by its non-regular variants. All these have been thoroughly studied during the past half century [1-11]. However, except for a single exception, regrettably mostly unknown, all results were limited to one dimension [12]. Yet physics happens mostly in two and three dimensions.

Recently we put forward a general recursive scheme to produce "paperfolding" structures in any dimension [13]. There we applied it in detail to two dimensions. Here we report progress in implementation of the algorithm to three and four dimensions.

\section{The recursion}

In order to keep this report self-contained we recapitulate the recursion rules. First we recall that the folds in dimension $d$ are cubes of dimension $d-1$. Thus, in three dimensions they are squares, in four dimensions three-dimensional cubes and so forth. Referring to Cartesian coordinate axes $x_{k}(k=1, \ldots, d)$ we always fold from the negative to the positive axis and cycle from 1 to $d$. In one dimension we fold always from left to right. Correspondingly, the regular paperfolding sequence $S$ is defined on the alphabet $\mathcal{A}_{2}=\{+,-\}$ by the recursion rule

$$
S(n+1)=\mathrm{m}^{\prime} S(n)+S(n), \quad S(0)=\emptyset,
$$

where $S(n)$ stands for the $n$-th generation of the sequence, $m^{\prime}$ denotes a mirror reflection with color change

*corresponding author; e-mail: shelomo.benabraham@gmail.com (in this case interchange $+\leftrightarrow-$ ) and $\emptyset$ stands for the empty chain. The first generation of the sequence thus becomes

$$
S(1)=+ \text {; }
$$

it is the "seed". Analogically, in higher dimensions the first generation serves as the seed.

In the case of three dimensions the first three folds orthogonal to the Cartesian axes 1 (or $x$ ), 2 (or $y$ ), 3 (or $z$ ), in that order, form the first generation, i.e. the seed. This is shown in Fig. 1.

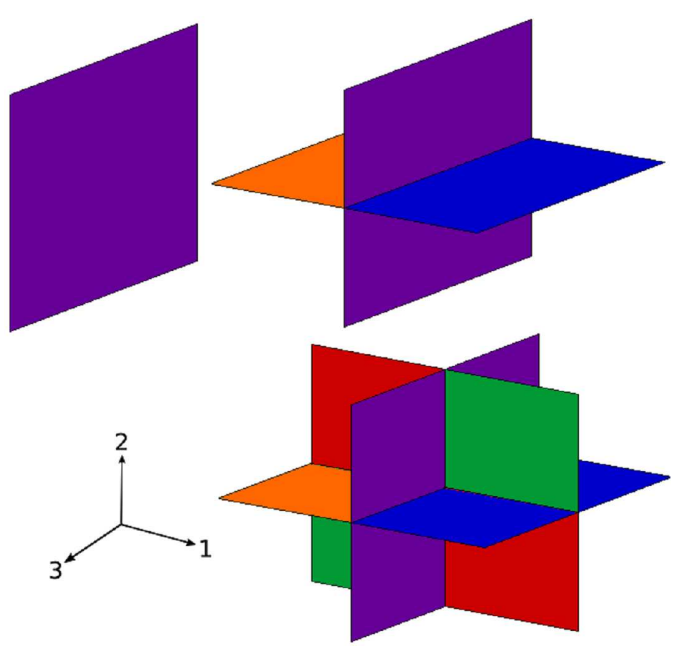

Fig. 1. The first three folds orthogonal to the axes $1(x), 2(y), 3(z)$ forming the first generation of the $3 \mathrm{D}$ structure - the seed.

The recursion rule for three dimensions is visualized in Fig. 2 showing schematically $S(n+1)$, i.e. generation $(n+1)$ of the structure in terms of the preceding generation $S(n) . S(n)$ is placed in the all plus sector (octant in $3 \mathrm{D})(+++)$. The content of all other sectors is generated by color mirrors $\mathrm{m}_{1}^{\prime}, \mathrm{m}_{2}^{\prime}, \mathrm{m}_{3}^{\prime}$ orthogonal to the Cartesian axes 1 ( or $x$ ), 2 ( or $y$ ), 3 (or $z$ ), respectively.

This can be formulated generally for arbitrary dimension $d$. In sector $\left(\sigma_{1}, \sigma_{2}, \ldots \sigma_{k}, \ldots, \sigma_{d}\right), \sigma_{k} \in\{+,-\}$ for each $\sigma_{k}=-$ there is a corresponding color mirror. Thus, 


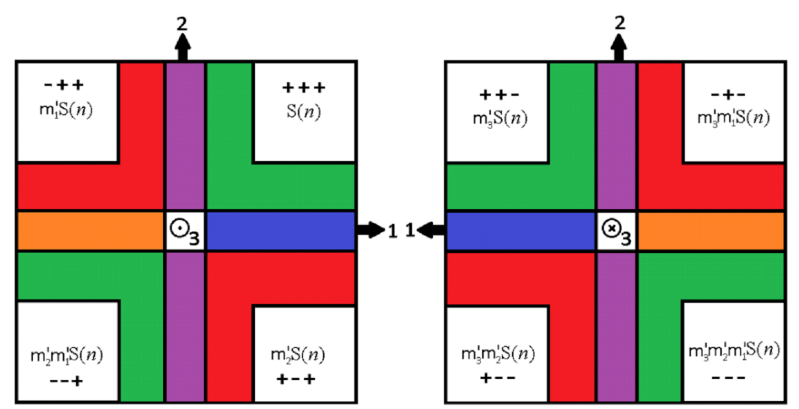

Fig. 2. The 3D recursion rule: $S(n+1)$ in terms of $S(n)$

for instance, in $4 \mathrm{D}$ we have explicitly

$$
\begin{aligned}
& (++++): S(n) \text {, } \\
& (-+++): m_{1}^{\prime} S(n),(+-++): m_{2}^{\prime} S(n), \\
& (++-+): m_{3}^{\prime} S(n),(+++-): m_{4}^{\prime} S(n) \text {, } \\
& \left(\text { - + +十): } m_{2}^{\prime} m_{1}^{\prime} S(n),(-+-+): m_{3}^{\prime} m_{1}^{\prime} S(n)\right. \text {, } \\
& (-++-): m_{4}^{\prime} m_{1}^{\prime} S(n) \text {, } \\
& \text { (+ - + ): } m_{3}^{\prime} m_{2}^{\prime} S(n),(+-+-): m_{4}^{\prime} m_{2}^{\prime} S(n) \text {, } \\
& (++--): m_{4}^{\prime} m_{3}^{\prime} S(n) \text {, } \\
& \left(\text { - + +十): } m_{1}^{\prime} S(n),(+-++): m_{2}^{\prime} S(n)\right. \text {, } \\
& (++-+): m_{3}^{\prime} S(n),(+++-): m_{4}^{\prime} S(n) \text {, } \\
& (----): m_{4}^{\prime} m_{3}^{\prime} m_{2}^{\prime} m_{1}^{\prime} S(n) \text {. }
\end{aligned}
$$

\section{Three dimensions}

The three-dimensional structure is a cubic honeycomb; the folds are two-dimensional squares, as mentioned earlier. This is shown in Fig. 3. It is not difficult to produce higher generations but for the sake of clarity the structure is displayed here only up to the fourth generation.

The alphabet is $\mathcal{A}_{6}=\left\{a_{1}, b_{1}, a_{2}, b_{2}, a_{3}, b_{3}\right\}$ where $a_{i}, b_{i}$ $(i=1,2,3)$ stand for positive and negative folds orthogonal to the coordinate axis $i$, respectively. Here they are color coded violet, yellow; blue, orange; green, red; respectively. The alphabet may be reduced by ignoring the orientation to $\mathcal{A}_{2}=\{+,-\}$ to produce a "black-and-white" version which may be preferable for physical applications (cf. [13]).

The tiles of the concomitant 3D tilings (cf. [13]) are octahedra or their topological deformations. These tilings will be dealt with elsewhere.

\section{Four dimensions}

The four-dimensional structure is a 4-cubic (also called hypercubic or tesseractic) honeycomb; the folds are three-dimensional cubes, as mentioned earlier.

In analogy to three dimensions the alphabet now becomes $\mathcal{A}_{8}=\left\{a_{1}, b_{1}, \ldots, a_{4}, b_{4}\right\}$ where $a_{i}, b_{i}(i=1,2,3,4)$

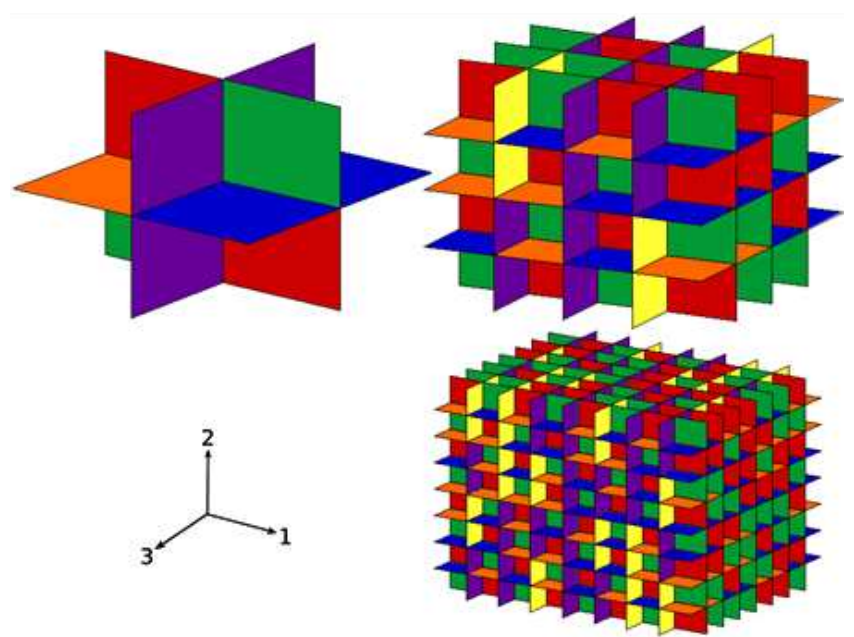

Fig. 3. The first three generations of the 3D PF structure.

stand for positive and negative folds orthogonal to the coordinate axis $i$, respectively. Here they are color coded violet, yellow; blue, orange; green, red; yellowish green, purple; respectively.

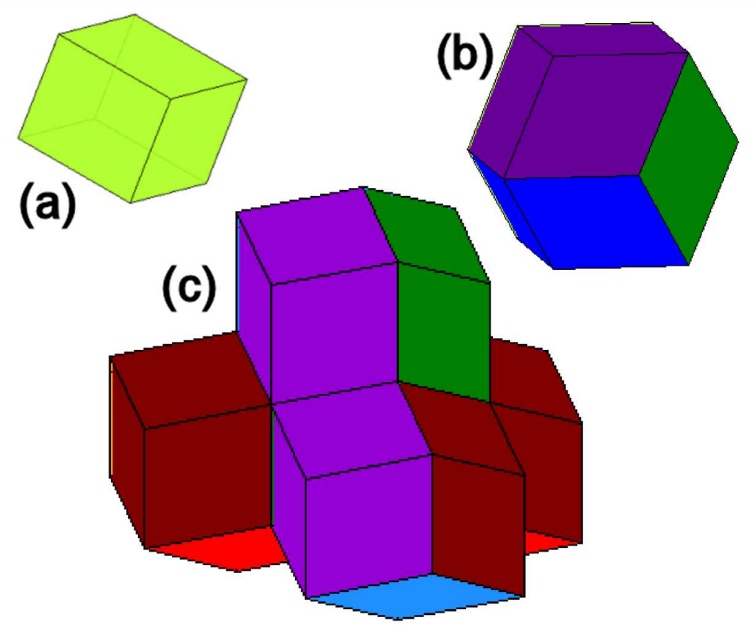

Fig. 4. (a) Oblate rhombohedron $-3 \mathrm{D}$ isometric projection of a fold in 4D (a 3-cube). (b) The $(++++)$ sector of the 4D PF structure. (c) Sectors $(-+++)$, $(+-++),(++-+),(+++-)$ of the $4 \mathrm{D}$ PF structure.

It is not quite easy to visualize a four-dimensional structure. The three-dimensional isometric (i.e. isogonal axonometric) projection of a 4-cube is a rhombic dodecahedron. The corresponding projection of a single fold (a 3-cube in 4D) is an oblate rhombohedron with face diagonals in the ratio $\sqrt{2}: 1$ (Fig. $4 \mathrm{a}$ ). Four rhombohedra meet in the center of the dodecahedron to form the projection of a $4 \mathrm{D}$ sector (hexadecant). Here we show the $(++++)$ sector (Fig. $4 \mathrm{~b})$ as well as its adjacent sectors $(-+++),(+-++),(++-+),(+++-)$ (Fig. 4c). 
A tile of the concomitant 4D tilings (cf. [13]) is a 16-cell (sometimes also called hexadecachoron or 4-orthoplex) or its topological deformations. These tilings will be dealt with elsewhere.

\section{The count}

In [13] we derived a general formula for the number of folds $|S(n)|$ in any generation $n$ of the structure $S$ in arbitrary dimension $d$ :

$$
|S(n)|=2^{(d-1) n}\left(2^{n}-1\right) d, \quad d, n \in N_{0} .
$$

Thus we have in three dimensions

$$
\begin{aligned}
& n=0:|S(0)|=2^{2 \times 0}\left(2^{0}-1\right) \times 3=0, \\
& n=1:|S(1)|=2^{2 \times 1}\left(2^{1}-1\right) \times 3=12, \\
& n=2:|S(2)|=2^{2 \times 2}\left(2^{2}-1\right) \times 3=144, \\
& n=3:|S(3)|=2^{2 \times 3}\left(2^{3}-1\right) \times 3=1344, \\
& n=4:|S(4)|=2^{2 \times 4}\left(2^{4}-1\right) \times 3=11420
\end{aligned}
$$

and so forth.

In four dimensions we have

$$
\begin{aligned}
& n=0:|S(0)|=2^{3 \times 0}\left(2^{0}-1\right) \times 4=0, \\
& n=1:|S(1)|=2^{3 \times 1}\left(2^{1}-1\right) \times 4=32, \\
& n=2:|S(2)|=2^{3 \times 2}\left(2^{2}-1\right) \times 4=768, \\
& n=3:|S(3)|=2^{3 \times 3}\left(2^{3}-1\right) \times 4=14336, \\
& n=4:|S(4)|=2^{3 \times 4}\left(2^{4}-1\right) \times 4=245960
\end{aligned}
$$

and so forth.

Clearly, the number of folds grows exponentially with both the dimension and the generation. It rapidly becomes staggering.

\section{Conclusion and outlook}

We have put forward a general recursive algorithm to generate "paperfolding" structures in arbitrary dimensions. Here we applied it to three and four dimensions. While 2D and 3D structures afford physical applications, structures in higher dimensions are mainly of mathematical interest. There remain some important open issues. It would be desirable to find a substitution (inflation) scheme as well as a closed formula for the symbolic complexity, at least in $2 \mathrm{D}$. The spectral properties of the structures are of prime importance. We conjecture that the Fourier spectrum is pure point (the Bragg peaks) even though we could not yet prove it. The numerical computations do indicate that. A strong argument in favor is that every row parallel to a Cartesian axis is a $1 \mathrm{D}$ paperfolding sequence and that is known to be pure point.

\section{References}

[1] M. Gardner, Sci. Am. 216(3), 124 (1967).

[2] M. Gardner, Sci. Am. 216(4), 116 (1967).

[3] M. Gardner, Sci. Am. 217(1), 112 (1967).

[4] C. Davis, D.E. Knuth, J. Recreational Math. 3, 66 (1970).

[5] C. Davis, D.E. Knuth, J. Recreational Math. 3, 133 (1970).

[6] M. Mendès-France, A.J. van der Poorten, M. Dekking, Math. Intelligencer 4, 130 (1982).

[7] M. Dekking, M. Mendès-France, A.J. van der Poorten, Math. Intelligencer 4, 173 (1982).

[8] M. Dekking, M. Mendès-France, A.J. van der Poorten, Math. Intelligencer 4, 190 (1982).

[9] M. Dekking, Theor. Comput. Sci. 414, 20 (2012).

[10] J.-P. Allouche, Bull. Austral. Math. Soc. 46, 23 (1992).

[11] J.-P. Allouche, J. Shallit, Automatic Sequences, Cambridge University Press, Cambridge 2003.

[12] O. Salon, J. Théor. Nombres Bordeaux 1, 1 (1989).

[13] S.I. Ben-Abraham, A. Quandt, D. Shapira, Acta Crystallogr. A 69, 123 (2013). 